\title{
DENSITY FLUCTUATIONS IN THE OSCILLATORY PHASE OF NONCLASSICAL INFLATON IN FRW UNIVERSE
}

\author{
K.K.Venkataratnam ${ }^{1}$ and P.K. Suresh 2 \\ School of Physics, University of Hyderabad, \\ Hyderabad-500046.India.
}

\begin{abstract}
Using coherent and squeezed state formalisms of quantum optics for a minimally coupled non-classical inflaton in the FRW mertic is studied, in semiclassical theory of gravity. The leading order solution for the semiclassical Einstein equations in the coherent, squeezed and squeezed vacuum states are obtained perturbatively and are exhibit powerlaw expansion behaviour. The validity of the semiclassical theory is examined in the squeezed vacuum state in the oscillatory phase of the inflaton. The semiclassical theory in the oscillatory phase of the non-classical inflaton holds only if the associated squeezing parameter is much less compared to unity. Quantum fluctuations of the inflaton is also examined in coherent and squeezed state formalisms.
\end{abstract}

\section{Introduction}

In standard cosmology, description of early universe is based on the Friedmann equations with scalar field(s). The Friedmann equations are based on classical gravity and assume that it valid even at very early stage of the universe also. However, quantum effects and quantum fluctuations of matter fields are expected to play a significant role in this regime, though quantum gravity effects are still negligible. To describe very early universe both gravity and matter fields are to be treated quantum mechanically, but at present there is no consistent quantum theory to describe gravity. Therefore, proper description of early universe with an appropriate cosmological model can be studied in terms of the semiclassical Friedmann equations, where gravity can be treated as classical with quantised matter field(s).

Recently, it has been found that non-classical state formalisms of quantum optics are found quite useful to deal with quantum effects in cosmology. The inflationary scenario has received much attention in semiclassical theory of gravity [1] [5. There are works in which quantum properties of the inflaton, which is responsible for the inflation, were investigated by many authors [6] [15. Such studies show that results obtained in classical gravity are quite different from those in semiclasical gravity. Though both classical and quantum inflaton in the oscillatory phase lead the same power law expansion, the correction to expansion does not show any oscillatory behaviour in semiclassical gravity in contrast to the oscillatory behaviour seen in classical gravity. It is to be noted that, the coherently oscillating inflaton suffers from particle production. Such studies reveal that quantum effects and quantum phenomena play an important role in inflation scenario and related issues.

Recently, we have studied 16] particle production during the oscillatory phase of the inflaton in squeezed and coherent state formalisms of quatum optics, in semiclassical theory of gravity. It is also interesting to study the validity of the semiclassical Einstein equation in the oscillatory phase of inflaton, because of quantum nature of the particle creation which may fluctuates the corresponding

\footnotetext{
${ }^{1}$ email:kkvratnam@yahoo.com

2 pkssp@uohyd.ernet.in
} 
energy density so widely. It is noted that the semiclassical theory of the Einstein's equations are valid only if the fluctuations of energy momentum tensor are not too large. The present work is to study the validity of the semiclassical Einstein equation in squeezed vacuum state during the oscillatory phase of inflaton. The validity of the equation can be studied with the help of the fluctuations of energy momentum tensor of the quantised inflaton.

The primary goal of the paper is to study quantum fluctuations and density fluctuations of a massive, minimal inflaton in the FRW universe in the semiclassical gravity by representing the inflaton in squeezed vacuum in the oscillatory phase of the inflaton. Hence to examine the validity of the Einstein equation in the oscillatory phase of the inflaton. Also we examine whether the solutions of the semiclassical equation, in the oscillatory phase of inflaton in coherent state, squeezed vacuum state and squeezed state, can lead to powerlaw expansion behaviour.

\section{Semiclassical Einstein equation and inflaton in FRW met- ric}

Most of the cosmological models are based on the classical gravity of Einstein equations with scalar field on the FRW metric. To study scalar field and the corresponding Friedmann equations at a deeper level, both background metric and the field are to be treated quantum mechanically. Since a consistent quantum theory of gravity is not available, in most of the cosmological models, the back ground metric is considered as classical (not quantised) and matter field as quantum. Such an approximation of the Einstein equation is known as semiclassical Einstein equation[17]. Therefore, in a semiclassical theory of gravity, Einstein equations take the following form (here onwards we use the unit system $\hbar=c=1$ and $\left.G=\frac{1}{m_{p}^{2}}\right)$ :

$$
G_{\mu \nu}=\frac{8 \pi}{m_{p}^{2}}\left\langle T_{\mu \nu}\right\rangle .
$$

Here, $G_{\mu \nu}$ is the Einstein tensor and $\left\langle T_{\mu \nu}\right\rangle$ is the expectation value of the energy-momentum tensor for matter field in a suitable quantum state under consideration with the quantum state satisfying the Schrödinger equation:

$$
i \frac{\partial}{\partial t}|\psi\rangle=\hat{H}|\psi\rangle .
$$

$|\psi\rangle$ denotes a quantum state and $\hat{H}$ is the Hamiltonian operator governing the quantum state. The semiclassical theory of gravity provides a description of the gravitational field of quantum systems with the expectation value of energy-momentum tensor as the source of gravity. Consider a flat Friedmann-Robertson-Walker spacetime

$$
d s^{2}=-d t^{2}+S^{2}(t)\left(d x^{2}+d y^{2}+d z^{2}\right) .
$$

Assume that a massive inflaton (a homogeneous scalar field) field, minimally coupled with gravity and its Lagrangian density can be written as

$$
\mathcal{L}=-\frac{1}{2} \sqrt{(-g)}\left(g^{\mu \nu} \partial_{\mu} \phi \partial_{\nu} \phi+m^{2} \phi^{2}\right) .
$$


The equation of motion of the inflaton is governed by the Klein-Gordon equation and can be written by using eqns (3) and (4) as

$$
\ddot{\phi}+3 \frac{\dot{S}(t)}{S(t)} \dot{\phi}+m^{2} \phi=0
$$

where overdot represents a derivative with respect to time. The inflaton field can be quantised by defining momentum conjugate to $\phi$ as $\pi=\frac{\partial L}{\partial \phi}$ and following the canonical quantisation procedure, the Hamiltonian for inflaton can be written as

$$
H=\frac{\pi^{2}}{2 S^{3}(t)}+\frac{1}{2} S^{3}(t) m^{2} \phi^{2} .
$$

Therefore, the temporal component of the energy-momentum tensor for the inflaton is obtained as

$$
T_{00}=S^{3}(t)\left(\frac{1}{2} \dot{\phi}^{2}+\frac{1}{2} m^{2} \phi^{2}\right) .
$$

\section{Coherent states and squeezed states}

Coherent and squeezed states 18 20 are important classes of quantum states, well-known in quantum optics. These states are being used as probes for studying the quantum effects in cosmology. Coherent states are considered as most classical, that can be generated from the vacuum state $|0\rangle$ by the action of displacement operator. In the present study, we use single mode coherent and squeezed states only. A single mode coherent state can be defined as

$$
|\alpha\rangle=D(\alpha)|0\rangle
$$

where $D(\alpha)$ is the single mode displacement operator, given by

$$
D(\alpha)=\exp \left(\alpha a^{\dagger}-\alpha^{*} a\right) .
$$

Here, $\alpha$ is a complex number and $a, a^{\dagger}$ are respectively the annihilation and creation operators, satisfying $\left[a, a^{\dagger}\right]=1$. The action of $a$ on the coherent state gives

$$
a|\alpha\rangle=\alpha|\alpha\rangle \text {. }
$$

The single mode displacement operator given by (9) satisfy the following property.

$$
D^{\dagger} a D=a+\alpha .
$$

A squeezed state is generated by the action of the squeezing operator on any coherent state is also on the vacuum state. Therefore, a single mode squeezed state is defined as

$$
|\alpha, \xi\rangle=Z(r, \varphi) D(\alpha)|0\rangle,
$$

with $Z(r, \varphi)$, the single mode squeezing operator given by,

$$
Z(r, \varphi)=\exp \frac{r}{2}\left(e^{-i \varphi} a^{2}-e^{i \varphi} a^{\dagger 2}\right) .
$$


Here, $r$ is the squeezing parameter, which determines the strength of the squeezing and $\varphi$ is the squeezing angle, which determines the distribution between conjugate variables, with $0 \leq r \leq \infty$ and $-\pi \leq \varphi \leq \pi$. The squeezing operator satisfy the following property

$$
Z^{\dagger} a Z=a \cosh r-a^{\dagger} e^{i \varphi} \sinh r .
$$

By setting $\alpha=0$ in (12), one obtains the squeezed vacuum state, and is defined as

$$
|\xi\rangle=Z(r, \varphi)|0\rangle \text {. }
$$

The squeezed vacuum state is a many-particle state and hence the resulting field may be called classical. However, the statistical properties of these states greatly differ from the coherent states and therefore, it is considered as highly non-classical having no analog in classical physics. In the case of coherent states, the variance of the conjugate variables are always equal to each other, while in a squeezed state one component of the noise is always squeezed with respect to the other. Therefore, in $(\mathrm{x}, \mathrm{p})$ plane, the noise for the coherent state can be described by a circle and for the squeezed state, it as an ellipse.

\section{Oscillatory phase of inflaton and power law expansion}

In this section, we consider a massive inflaton minimally coupled to a spatially flat FRW metric and study inflaton in nonclassical states. First, we consider the inflaton in a nonclassical state which is closest to the classical state i.e., the coherent states and then extend it to the squeezed state representation of the inflaton.

As mentioned earlier, we consider a massive inflaton, minimally coupled to a spatially flat FRW universe with the metric (3). Therefore, the time-time component of the classical gravity is now the classical Einstein equation

$$
\left(\frac{\dot{S}}{S}\right)^{2}=\frac{8 \pi}{3 m_{p}^{2}} \frac{T_{00}}{S^{3}(t)},
$$

where $T_{00}$ is the energy density of the inflaton, given by (7).

In the cosmological context, the classical Einstein equation (16) means that the Hubble param-

eter, $H=\frac{\dot{S}}{S}$, is determined by the energy density of the dynamically evolving inflaton as described by (16). In the semiclassical theory, the Friedmann equation can be written as:

$$
\left(\frac{\dot{S}}{S}\right)^{2}=\frac{8 \pi}{3 m_{p}^{2}} \frac{1}{S^{3}(t)}\left\langle\hat{H}_{m}\right\rangle,
$$

where $\left\langle\hat{H}_{m}\right\rangle$ represent the expectation value of the Hamiltonian of the scalar field in a quantum state under consideration.

The massive minimal inflaton in the flat FRW universe can be described by the time dependent harmonic oscillator with the Hamiltonian given by eq(6). For the semiclassical Friedmann equation (17), we have to find the expectation value of the Hamiltonian in a given quantum state. Then the eigenstates of the Hamiltonian are the Fock states:

$$
\hat{a^{\dagger}}(t) \hat{a}(t)|n, \phi, t\rangle=n|n, \phi, t\rangle,
$$


where $a$ and $a^{\dagger}$ are the annihilation and creation operators obeying boson commutation relations $\left[a, a^{\dagger}\right]=1$, the other combinations being zero. These can respectively be written as:

$$
\begin{aligned}
\hat{a}(t) & =\phi^{*}(t) \hat{\pi}-S^{3} \dot{\phi}^{*}(t) \hat{\phi}, \\
\hat{a^{\dagger}}(t) & =\phi(t) \hat{\pi}-S^{3} \dot{\phi}(t) \hat{\phi} .
\end{aligned}
$$

The expectation value of the Hamiltonian can be calculated in the number state by using (17), (18) and (19) and hence, the semiclassical Friedmann equation (17), in the number state, can be written as

$$
\left(\frac{\dot{S}(t)}{S(t)}\right)^{2}=\frac{8 \pi}{3 m_{p}^{2}}\left[\left(n+\frac{1}{2}\right)\left(\dot{\phi}^{*} \dot{\phi}+m^{2} \phi^{*} \phi\right)\right] .
$$

In (20), $\phi$ and $\phi^{*}$ satisfy (5) and the Wronskian condition

$$
S^{3}(t)\left(\dot{\phi}^{*}(t) \phi(t)-\phi^{*}(t) \dot{\phi}(t)\right)=i .
$$

The Wronskian and the boundary conditions, fix the normalisation constants of the two independent solutions.

As an alternative to the $n$ representation of the inflaton, we next consider the inflaton in the coherent and the squeezed states formalisms and hence the semiclassical Einstein equation can be expressed in terms of their respective parameters.

Using (8-11), (17) and (19) the semiclassical Einstein equation can be written for the coherent state as

$$
\left(\frac{\dot{S}(t)}{S(t)}\right)^{2}=\frac{8 \pi}{3 m_{p}^{2}}\left[\left(|\alpha|^{2}+\frac{1}{2}\right)\left(\dot{\phi}^{*} \dot{\phi}+m^{2} \phi^{*} \phi\right)-\frac{1}{2} \alpha^{* 2}\left(\dot{\phi}^{* 2}+m^{2} \phi^{* 2}\right)-\frac{1}{2} \alpha^{2}\left(\dot{\phi}^{2}+m^{2} \phi^{2}\right)\right] .
$$

By using (12-14), (17) and (19), the semiclassical equation for the squeezed vacuum state is obtained as

$$
\begin{aligned}
\left(\frac{\dot{S}(t)}{S(t)}\right)^{2}= & \frac{8 \pi}{3 m_{p}^{2}}\left[\left(\sinh ^{2} r+\frac{1}{2}\right)\left(\dot{\phi}^{*} \dot{\phi}+m^{2} \phi^{*} \phi\right)\right. \\
& \left.+\frac{\sinh 2 r}{4}\left[e^{-i \varphi}\left(\dot{\phi}^{* 2}+m^{2} \phi^{* 2}\right)+e^{i \varphi}\left(\dot{\phi}^{2}+m^{2} \phi^{2}\right)\right]\right] .
\end{aligned}
$$

Similarly, the semiclassical equation for a squeezed state is obtained as

$$
\begin{aligned}
\left(\frac{\dot{S}(t)}{S(t)}\right)^{2}= & \frac{8 \pi}{3 m_{p}^{2}}\left[\left(\sinh ^{2} r+\frac{1}{2}+|\alpha|^{2}\right)\left(\dot{\phi}^{*} \dot{\phi}+m^{2} \phi^{*} \phi\right)\right. \\
& +\left(\frac{e^{-i \varphi} \sinh r \cosh r-\alpha^{* 2}}{2}\right)\left(\dot{\phi}^{* 2}+m^{2} \phi^{* 2}\right) \\
& \left.+\left(\frac{e^{i \varphi} \sinh r \cosh r-\alpha^{2}}{2}\right)\left(\dot{\phi}^{2}+m^{2} \phi^{2}\right)\right] .
\end{aligned}
$$


Our next task is to solve these semiclassical Einstein equations for which we transform the solution in the following form

$$
\phi(t)=\frac{1}{S^{\frac{3}{2}}} \psi(t),
$$

thereby obtaining

$$
\ddot{\psi}(t)+\left(m^{2}-\frac{3}{4}\left(\frac{\dot{S}(t)}{S(t)}\right)^{2}-\frac{3}{2} \frac{\ddot{S}(t)}{S(t)}\right) \psi(t)=0
$$

Now we focus on the oscillatory phase of the inflaton after inflation. In the parameter region satisfying the inequality

$$
m^{2}>\frac{3 \dot{S}^{2}}{4 S^{2}}+\frac{3 \ddot{S}}{2 S},
$$

the inflaton has an oscillatory solution of the form

$$
\psi(t)=\frac{1}{\sqrt{2 w(t)}} \exp \left(-i \int w(t) d t\right),
$$

with

$$
w^{2}(t)=m^{2}-\frac{3}{4}\left(\frac{\dot{S}(t)}{S(t)}\right)^{2}-\frac{3}{2} \frac{\ddot{S}(t)}{S(t)}+\frac{3}{4}\left(\frac{\dot{w}(t)}{w(t)}\right)^{2}-\frac{1}{2} \frac{\ddot{w}(t)}{w(t)} .
$$

Using (25),(28) and (29) in (20), we get

$$
\left(\frac{\dot{S}(t)}{S(t)}\right)^{2}=\left[\frac{4 \pi}{3 m_{p}^{2}}\left(n+\frac{1}{2}\right) \frac{1}{S^{3}} \frac{1}{w(t)}\left(w^{2}(t)+m^{2}+\frac{1}{4}\left(\frac{\dot{w}(t)}{w(t)}+\frac{3 \dot{S}(t)}{S(t)}\right)^{2}\right)\right] .
$$

Therefore

$$
S(t)=\left[\frac{4 \pi}{3 m_{p}^{2}}\left(n+\frac{1}{2}\right) \frac{1}{w(t)\left(\frac{\dot{S}(t)}{S(t)}\right)^{2}}\left(w^{2}(t)+m^{2}+\frac{1}{4}\left(\frac{\dot{w}(t)}{w(t)}+\frac{3 \dot{S}(t)}{S(t)}\right)^{2}\right)\right]^{\frac{1}{3}} .
$$

We solve (31) using the following approximation ansatzs

$$
w_{0}(t)=m ; \quad S_{0}(t)=S_{0} t^{\frac{2}{3}} .
$$

Thus the next order perturbative solution is obtained for the number state as

$$
S_{1}(t)=\left[\frac{6 \pi}{m_{p}^{2}}\left(n+\frac{1}{2}\right) m t^{2}\left(1+\frac{1}{2 m^{2} t^{2}}\right)\right]^{\frac{1}{3}} .
$$

Similarly the perturbative solution for the coherent state can be computed and hence the scale factor 
for the coherent state is obtained as

$$
\begin{aligned}
S(t)= & {\left[\frac { 8 \pi } { 3 m _ { p } ^ { 2 } ( \frac { \dot { S } ( t ) } { S ( t ) } ) ^ { 2 } } \left[\frac{\left(|\alpha|^{2}+\frac{1}{2}\right)\left(A+w^{2}(t)+m^{2}\right)}{2 w(t)}\right.\right.} \\
& -\frac{1}{2} \frac{\alpha^{* 2} \exp \left(2 i \int w(t) d t\right)}{2 w(t)}\left(A-w^{2}(t)-i w(t)\left[3 \frac{\dot{S}(t)}{S(t)}+\frac{\dot{w}(t)}{w(t)}\right]+m^{2}\right) \\
& \left.\left.-\frac{1}{2} \frac{\alpha^{2} \exp \left(-2 i \int w(t) d t\right)}{2 w(t)}\left(A-w^{2}(t)+i w(t)\left[3 \frac{\dot{S}(t)}{S(t)}+\frac{\dot{w}(t)}{w(t)}\right]+m^{2}\right)\right]\right]^{\frac{1}{3}},
\end{aligned}
$$

where

$$
A=\frac{1}{4}\left(\frac{\dot{w}}{w}+3 \frac{\dot{S}}{S}\right)^{2} .
$$

Applying the approximation ansatzs (32), then the next order perturbative solutions for the coherent state is obtained as

$$
\begin{aligned}
S_{1}(t)= & {\left[\frac { 6 \pi t ^ { 2 } } { m _ { p } ^ { 2 } } \left[\frac{\left(|\alpha|^{2}+\frac{1}{2}\right)\left(\frac{1}{t^{2}}+2 m^{2}\right)}{2 m}\right.\right.} \\
& -\frac{1}{2} \frac{\alpha^{* 2} \exp \left(2 i \int m d t\right)}{2 m}\left(\frac{1}{t^{2}}-\frac{2 i m}{t}\right) \\
& \left.\left.-\frac{1}{2} \frac{\alpha^{2} \exp \left(-2 i \int m d t\right)}{2 m}\left(\frac{1}{t^{2}}+\frac{2 i m}{t}\right)\right]\right]^{\frac{1}{3}} .
\end{aligned}
$$

Using $\alpha=e^{i \theta} \alpha$, we get

$$
\begin{aligned}
S_{1}(t)_{c s}= & {\left[\frac{6 \pi}{m_{p}^{2}}\left(|\alpha|^{2}+\frac{1}{2}\right) m t^{2}\left(1+\frac{1}{2 m^{2} t^{2}}\right)-\frac{3 \alpha^{2}}{m} \frac{\pi t^{2}}{m_{p}^{2}}\right.} \\
& \left.\times\left[\frac{\cos 2(\theta-m t)}{t^{2}}-\frac{2 m}{t} \sin 2(\theta-m t)\right]\right]^{\frac{1}{3}} .
\end{aligned}
$$

Using the approximation ansatzs (32) and the next order perturbative solution for the squeezed vacuum state becomes,

$$
\begin{aligned}
S_{1}(t)_{s v s}= & {\left[\frac{6 \pi}{m_{p}^{2}}\left(\sinh ^{2} r+\frac{1}{2}\right) m t^{2}\left(1+\frac{1}{2 m^{2} t^{2}}\right)+\frac{6 \pi t^{2}}{m_{p}^{2}} \frac{\sinh 2 r}{4}\right.} \\
& \left.\times\left[\frac{\cos (\varphi-2 m t)}{m t^{2}}-\frac{2}{t} \sin (\varphi-2 m t)\right]\right]^{\frac{1}{3}} .
\end{aligned}
$$


Similarly, we get the next order perturbative solution for the squeezed state as

$$
\begin{aligned}
S_{1}(t)_{s s}= & {\left[\frac{6 \pi}{m_{p}^{2}}\left(\sinh ^{2} r+|\alpha|^{2}+\frac{1}{2}\right) m t^{2}\left(1+\frac{1}{2 m^{2} t^{2}}\right)+\frac{6 \pi t^{2}}{m_{p}^{2}} \frac{\sinh 2 r}{4}\right.} \\
& \times\left[\frac{\cos (\varphi-2 m t)}{m t^{2}}-\frac{2}{t} \sin (\varphi-2 m t)\right] \\
& \left.-\frac{3 \alpha^{2}}{m} \frac{\pi t^{2}}{m_{p}^{2}}\left[\frac{\cos 2(\theta-m t)}{t^{2}}-\frac{2 m}{t} \sin 2(\theta-m t)\right]\right]^{\frac{1}{3}} .
\end{aligned}
$$

From (36), (37) and (38), it follows that $S_{1}(t)_{c s} \sim t^{\frac{2}{3}}, S_{1}(t)_{s v s} \sim t^{\frac{2}{3}}$ and $S_{1}(t)_{s s} \sim t^{\frac{2}{3}}$

Therefore, in the oscillating phase of the inflaton in coherent, squeezed vacuum and squeezed states, the approximate leading solution to the semiclassical Einstein equation has the same powerlaw expansion.

\section{Density fluctuations and validity of semiclassical theory}

The semiclassical theory of gravity valid only if the fluctuations in the energy momentum tensor are not large. Thus the validity of the semiclassical theory can be studied with the help of the energy momentum tensor of the inflaton in a suitable quantum states under the consideration. We examine the validity of the Einstein equation in squeezed vacuum state which is purely exhibit quatum features. The checking of the validity of the Einstein equation can be done with the help a quantity defined below in terms of the energy momentum tensor as

$$
\Delta=\left|\left\langle: T_{\mu \nu}^{2}:\right\rangle-\left\langle: T_{\mu \nu}:\right\rangle^{2}\right|
$$

Where $\left\langle: T_{\mu \nu}^{2}:\right\rangle$ is the expectation value of the squared energy momentum tensor of a scalar field in a suitable quatum state and $\left\langle: T_{\mu \nu}:\right\rangle^{2}$ is its square of the expectation value. The meaning of : : is that the expectation values are to be computed with respect to the normal ordering of the scalar field. If the $\Delta$ is more than one then the corresponding semiclassical Einstein equation does not hold in that particular quantum state. For the sake of simplicity of the study, we focus on the temporal component of the energy momentum tensor with single mode of the inflaton and investigate the validity of the semiclassical theory in squeezed vacuum state. Thus we need consider only the density fluctuations which can be written in squeezed vacuum state as

$$
\Delta_{\text {svs }}=\left|<: T_{00}^{2}:>_{\text {svs }}-<: T_{00}:>_{\text {svs }}^{2}\right| .
$$

The first term of eqn(40) can be obtained by squaring eq(7) and taking the expectation value in squeezed vacuum state as

$$
\begin{aligned}
<: T_{00}^{2}:>_{\text {svs }} & =\frac{1}{4 S^{6}(t)}<: \hat{\pi}^{4}:>_{\text {svs }}+\frac{m^{2}}{4}<: \hat{\pi}^{2} \hat{\phi}^{2}:>_{\text {svs }} \\
& +\frac{m^{2}}{4}<: \hat{\phi}^{2} \hat{\pi}^{2}:>_{\text {svs }}+\frac{m^{4}}{4} S^{6}(t)<: \hat{\phi}^{4}:>_{\text {svs }} .
\end{aligned}
$$


Using the eqns (14),(15) and apply the approximations ansatz then, $<: \hat{\pi}^{4}:>_{\text {svs }},<: \hat{\pi}^{2} \hat{\phi}^{2}:>_{\text {svs }},<:$ $\hat{\phi}^{2} \hat{\pi}^{2}:>_{\text {svs }}$ and $<: \hat{\phi}^{4}:>_{\text {svs }}$ can be calculated and are respectively obtained as follows

$$
\begin{aligned}
<: \hat{\pi}^{4}:>_{\text {svs }}= & S_{0}^{6}\left[\frac { 1 } { 4 m ^ { 2 } } \left\{3+6 \cosh ^{2} r \sinh ^{2} r+24 \sinh ^{3} r \cosh r\right.\right. \\
& \left.\left.+12\left(\sinh ^{2} r+\sinh ^{4} r+\cosh r \sinh r\right)\right\}\right] \\
<: \hat{\pi}^{2} \hat{\phi}^{2}:>_{\text {svs }}= & \frac{1}{4 m^{2} t^{2}}\left\{3+6 \cosh ^{2} r \sinh ^{2} r+24 \sinh ^{3} r \cosh r\right. \\
& \left.+12\left(\sinh ^{2} r+\sinh ^{4} r+\cosh r \sinh r\right)\right\} \\
<: \hat{\phi}^{2} \hat{\pi}^{2}:>_{\text {svs }}= & \frac{1}{4 m^{2} t^{2}}\left\{3+6 \cosh ^{2} r \sinh ^{2} r+24 \sinh ^{3} r \cosh r\right. \\
& \left.+12\left(\sinh ^{2} r+\sinh ^{4} r+\sinh r \cosh r\right)\right\} \\
<: \hat{\phi}^{4}:>_{\text {svs }}= & \frac{1}{4 S_{0}^{6} t^{4} m^{2}}\left\{3+6 \cosh ^{2} r \sinh { }^{2} r+24 \cosh r \sinh { }^{3} r\right. \\
& \left.+12\left(\sinh ^{2} r+\sinh ^{4} r+\sinh r \cosh r\right)\right\} .
\end{aligned}
$$

Thus

$$
\begin{aligned}
<: T_{00}^{2}:>_{s v s} & =\left(\frac{1}{16 m^{2} t^{4}}+\frac{1}{8 t^{2}}+\frac{m^{2}}{16}\right)\left[3+6 \cosh ^{2} r \sinh ^{2} r+24 \sinh ^{3} r \cosh r\right. \\
& \left.+12\left(\sinh ^{2}+\sinh ^{4} r+\cosh r \sinh r\right)\right] .
\end{aligned}
$$

By calculating the expectation value of normal ordered time-time component of energy momentum tensor of the inflaton in squeezed vacuum state and then square the result which leads to

$$
\begin{aligned}
<: T_{00}:>_{\text {svs }}^{2}= & S^{6}\left[\left(\sinh ^{4} r+\frac{1}{4}+\sinh ^{2} r\right)\left(\dot{\phi}^{* 2} \dot{\phi}^{2}+2 m^{2} \dot{\phi}^{*} \dot{\phi} \phi^{*} \phi+m^{4} \phi^{* 2} \phi^{2}\right)\right. \\
& +\frac{1}{4}\left(e^{-2 i \varphi} \sinh ^{2} r \cosh ^{2} r\right)\left(\dot{\phi}^{* 4}+2 m^{2} \dot{\phi}^{* 2} \phi^{* 2}+m^{4} \phi^{* 4}\right) \\
& +\frac{1}{4} e^{2 i \varphi} \sinh ^{2} r \cosh ^{2} r\left(\dot{\phi}^{4}+m^{4} \phi^{4}+2 m^{2} \dot{\phi}^{2} \phi^{2}\right) \\
& +\left(\sinh ^{2} r+\frac{1}{2}\right) e^{-i \varphi} \sinh r \cosh r\left(\dot{\phi} \dot{\phi}^{* 3}+m^{2} \phi^{*} \phi \dot{\phi}^{* 2}\right. \\
& \left.+m^{2} \dot{\phi}^{*} \dot{\phi} \phi^{* 2}+m^{4} \phi \phi^{* 3}\right) \\
& +\frac{1}{2} \sinh ^{2} r \cosh { }^{2} r\left(\dot{\phi}^{* 2} \dot{\phi}^{2}+m^{2} \dot{\phi}^{* 2} \phi^{2}+m^{2} \phi^{* 2} \dot{\phi}^{2}+m^{4} \phi^{* 2} \phi^{2}\right) \\
& +e^{i \varphi} \sinh ^{2} \cosh r\left(\sinh ^{2} r+\frac{1}{2}\right)\left(\dot{\phi}^{2} \dot{\phi}^{*} \dot{\phi}+m^{2} \phi^{2} \dot{\phi}^{*} \dot{\phi}\right. \\
& \left.\left.+m^{2} \dot{\phi}^{2} \phi^{*} \phi+m^{4} \phi^{2} \phi^{*} \phi\right)\right] .
\end{aligned}
$$




$\begin{array}{cccccccc}r & \Delta_{\text {svs }} & r & \Delta_{\text {svs }} & r & \Delta_{\text {svs }} & r & \Delta_{\text {svs }} \\ 0.001 & 0.4970 & 0.010 & 0.4699 & 0.100 & 0.1869 & 1.100 & 32.5227 \\ 0.002 & 0.4940 & 0.020 & 0.4397 & 0.200 & 0.1872 & 1.200 & 48.7983 \\ 0.003 & 0.4910 & 0.030 & 0.4093 & 0.300 & 0.6828 & 1.300 & 73.0775 \\ 0.004 & 0.4880 & 0.040 & 0.3787 & 0.400 & 1.3803 & 1.400 & 109.2970 \\ 0.005 & 0.4850 & 0.050 & 0.3477 & 0.500 & 2.3928 & 1.500 & 163.3297 \\ 0.006 & 0.4820 & 0.060 & 0.3165 & 0.600 & 3.8845 & 1.600 & 243.9366 \\ 0.007 & 0.4790 & 0.070 & 0.2848 & 0.700 & 6.0972 & 1.700 & 364.1878 \\ 0.008 & 0.4760 & 0.080 & 0.2527 & 0.800 & 9.3898 & 1.800 & 543.5814 \\ 0.009 & 0.4730 & 0.090 & 0.2200 & 0.900 & 14.2961 & 1.900 & 811.2051 \\ 0.010 & 0.4699 & 0.100 & 0.1869 & 1.000 & 21.6117 & 2.000 & 1210.4528\end{array}$

Table I Numerical values of $\Delta_{\text {svs }}$ for squeezing parameter $r$ much smaller and larger compared to unity

Using equations (25) and (28) apply the approximation ansatz eqn(32) in (47) we get

$$
\begin{aligned}
<: T_{00}:>_{\text {svs }}^{2}= & \left(\frac{1}{4 m^{2} t^{4}}+\frac{1}{2 t^{2}}+\frac{m^{2}}{4}\right)\left(\frac{1}{4}+\sinh ^{4} r+\sinh ^{2} r+\sinh ^{2} r \cosh ^{2} r\right. \\
& \left.+2 \sinh ^{3} r \cosh r+\sinh r \cosh r\right) .
\end{aligned}
$$

Substituting equations(46) and (48) in eqn (40), then

$$
\begin{aligned}
\Delta_{\text {svs }}= & \left(\frac{1}{16 m^{2} t^{4}}+\frac{1}{8 t^{2}}+\frac{m^{2}}{16}\right)\left[2+2 \cosh ^{2} r \sinh ^{2} r+16 \sinh ^{3} r \cosh r\right. \\
& \left.+8\left(\sinh ^{2} r+\sinh ^{4} r+\cosh r \sinh r\right)\right] .
\end{aligned}
$$

In order to examine the validity of the semiclassical equation, we study $\Delta_{\text {svs }}$ numerically with the associated squeezing parameter values which are much smaller and larger compared to unity and corresponding results are tabulated in Table I.

In the large squeezing limit compared to much smaller value of the squeezing parameter, the equation(49) becomes

$$
\Delta_{s v s} \approx\left(\frac{1}{16 m^{2} t^{4}}+\frac{1}{8 t^{2}}+\frac{m^{2}}{16}\right)\left[\frac{1}{2}+\frac{13}{8} e^{4 r}-e^{2 r}\right] .
$$

From these analysis, it is clear that the semiclassical Einstein equation in the oscillatory phase of the non-classical inflaton in squeezed vacuum state formalism is valid only provided the corresponding squeezing parameter is much smaller compared to unity. The density fluctuations arise because of the particle creation due to the quantum nature of the squeezed vacuum state during the oscillatory phase of the inflaton, in the semiclassical gravity. 


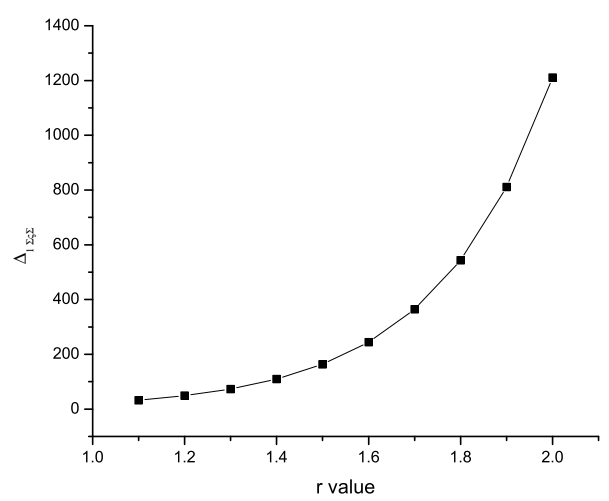

Figure 1: Behaviour of $\Delta_{\text {svs }}$ for large squeezing limit compared to unity for the calculated values of $\Delta_{\text {svs }}$ verses squeezing parameter $\mathrm{r}$

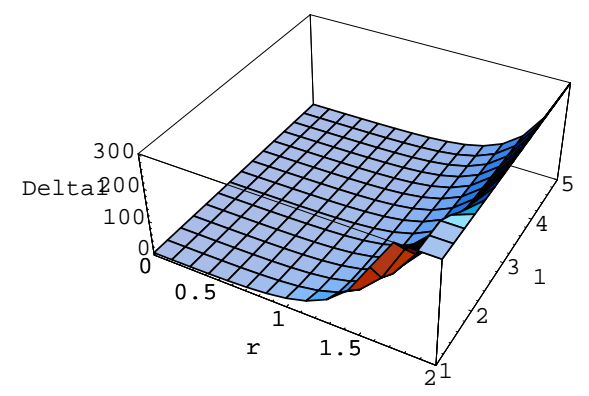

Figure 2: $\Delta_{\text {svs }}$ in squeezed vacuum for $r$ and $t$ 


\section{Quantum fluctuations}

In this section we discuss quantum fluctuations for the coherently oscillating inflaton in semiclassical theory of gravity. The quantum fluctuations in coherent and squeezed states representation of the inflaton can be studied by using dispersion relations of $\phi$ and $\pi$, which are, respectively, given by

$$
(\Delta \phi)^{2}=\left\langle\hat{\phi}^{2}\right\rangle-\langle\hat{\phi}\rangle^{2}
$$

and

$$
(\Delta \pi)^{2}=\left\langle\hat{\pi}^{2}\right\rangle-\langle\hat{\pi}\rangle^{2} .
$$

Here, $\left\langle\hat{\phi}^{2}\right\rangle,\left\langle\hat{\pi}^{2}\right\rangle,\langle\hat{\phi}\rangle$ and $\langle\hat{\pi}\rangle$ are respectively the squared expectation values and expectation values of $\phi$ and $\pi$ in a given quantum state under consideration.

First, we consider quantum fluctuations of inflaton in coherent state. Using (8-11) and (19), it follows that

$$
(\Delta \phi)_{c s}=\sqrt{\phi^{*} \phi}
$$

and

$$
(\Delta \pi)_{c s}=S^{3} \sqrt{\dot{\phi}^{*} \dot{\phi}}
$$

Using eqns( 27) and (28) in the equations(53) and (54) respectively and then applying the approximation ansatz (32) we get the dispersion relations in coherent state as

$$
(\Delta \phi)_{c s}=\sqrt{\frac{1}{S_{0}^{3} t^{2}} \frac{1}{2 m}},
$$

and

$$
(\Delta \pi)_{c s}=S_{0}^{3} t^{2} \sqrt{\frac{1}{S_{0}^{3} t^{2}} \frac{1}{2 m}}\left(\frac{1}{t^{2}}+m^{2}\right) .
$$

In the limit $m t>>1$, the above two expressions gives the dispersion relation in coherent states as

$$
(\Delta \phi)_{c s}(\Delta \pi)_{c s}=\frac{1}{2} \sqrt{\left(1+\frac{1}{m^{2} t^{2}}\right)} .
$$

The dispersion relations in squeezed vacuum state can be calculated by the same procedure and is obtained in the limit $m t>>1$, as

$$
\begin{aligned}
(\Delta \phi)_{s v s}(\Delta \pi)_{s v s} & =\frac{1}{2 m}\left[\left[\left(2 \sinh ^{2} r+1\right)+2 \sinh r \cosh r \cos (\varphi-2 m t)\right]\right. \\
& \times\left(2 \sinh ^{2} r+1\right)\left(\frac{1}{t^{2}}+m^{2}\right)+\sinh r \cosh r \\
& \left.\times\left[\frac{2}{t^{2}} \cos (\varphi-2 m t)-\frac{4 m}{t} \sin (\varphi-2 m t)-2 m^{2} \cos (\varphi-2 m t)\right]\right]^{\frac{1}{2}} .
\end{aligned}
$$


Similarly for the squeezed state, we get

$$
\begin{aligned}
(\Delta \phi)_{s s}(\Delta \pi)_{s s} & =\frac{1}{2 m}\left[\left[\left(2 \sinh ^{2} r+1\right)+2 \sinh r \cosh r \cos (\varphi-2 m t)\right]\right. \\
& \times\left(2 \sinh ^{2} r+1\right)\left(\frac{1}{t^{2}}+m^{2}\right)+\sinh r \cosh r \\
& \left.\times\left[\frac{2}{t^{2}} \cos (\varphi-2 m t)-\frac{4 m}{t} \sin (\varphi-2 m t)-2 m^{2} \cos (\varphi-2 m t)\right]\right]^{\frac{1}{2}} .
\end{aligned}
$$

\section{Discussions and Conclusions}

Quantum effects of matter field can play a significant role in the early universe. In order to study quantum effects in an early universe scenario, both background metric and matter field under consideration are to be treated quantum mechanically. Although, there are have been attempts to formulate the quantum theory of gravity, still we do not have a consistent quantum theory of gravity. Therefore appropriate conditions, quantum effects in various cosmological problems can be studied in a semiclassical approach. In this approach, the background metric is treated as classical and the matter field as quantum mechanical. In the present work, we studied a homogeneous massive scalar field minimally coupled to the Friedmann-Robertson-Walker universe in the context of semiclassical theory of gravity. The quantum effect of the scalar field is studied by representing the inflaton in various nonclassical states, such as coherent and squeezed states. We particularly addressed the oscillatory phase of the inflaton after inflation in coherent and squeezed states formalisms.

Approximate leading solutions to the Einstein equation, in coherent and squeezed state formalisms are obtained perturbatively. It is found that the solution for coherent state depends on the coherent state parameter and its phase factor, while the solution for squeezed states, depends on the associated squeezing parameter and squeezing angle. It can be concluded that in the oscillatory phase of the inflaton, in coherent and squeezed state representations, the approximate leading solution to the semiclassical Einstein equation has the same power-law expansion, as the classical Einstein equation. However, in the coherent and squeezed states formalisms, the correction terms do not oscillate in contrast with the classical gravity.

We studied the quantum fluctuations due to the coherently oscillating inflaton after the inflation in coherent state and squeezed state formalisms, in the frame work of semiclassical theory of gravity. The quantum fluctuations of the inflaton studied in terms of dispersion relations of $\phi$ and $\pi$ and the study of the coherent state shows that the dispersion relations of the inflaton is inversely proportional to $t$. Also the study reveals that the uncertainty relation for coherent state does not depend on the coherent state parameter. While the uncertainty relation calculated for squeezed vacuum state shows that the relation depends on the associated squeezing parameter and the squeezing angle.

We studied density fluctuations in squeezed vacuum state formalism. It is found the analytical result for density fluctuations and hence examined the validity of the semiclassical Einstein equation. The validity of the semiclassical Einstein equation is studied in terms of the energy density of the inflaton field in the oscillating phase of inflaton. It is observed that the semiclassical equation hold only when the associated squeezing parameter is much less than one otherwise the density fluctuates so widely because of the particle creation due to the quantum nature of the squeezed vacuum state during the oscillatory phase of the inflaton. 


\section{References}

[1] B. K. Berger, Phys.Rev.D23, 1250 (1981).

[2] S. Habib, Phys.Rev.D46, 2408 (1992).

[3] A. Linde, D. Linde and A Mezhlumian, Phys.Rev.D49, 1783 (1994).

[4] B. Dongsu Bak et.al., Phys.Rev.D59, 027301 (1998).

[5] S. P. Kim and D. N. Page, J.Kor.Phys.Soc.35, S660 (1999).

[6] M. Gasperini and M. Giovannini, Phys.Rev.D50, 4807 (1994).

[7] B. L. Hu, G. Kang and A. L. Matacz, Int.J.Mod.Phys.A9, 991 (1994).

[8] P. K. Suresh, V. C. Kuriakose and K. Babu Joseph, Int.J.Mod.Phys. D6, 771 (1995).

[9] P. K. Suresh and V. C. Kuriakose, Mod.Phys.Lett.A13, 165 (1998).

[10] A. L. Matacz, P. C. W. Davies and A. C. Ottewill, Phys. Rev.D47, 1557 (1993).

[11] A. Albrecht et.al., Phys.Rev.D50, 4807 (1994).

[12] R. Brandenberger, V. Mukhanov and T. Prokopec, Phys.Rev.Lett. 69, 3606 (1992).

[13] R. Brandenberger, V. Mukhanov and T.Prokopec, Phys.Rev.D48, 2443 (1993).

[14] L. P. Grishchuk and Y. V. Sidorov, Phys.Rev.D42, 3413 (1993).

[15] C. Kuo and L. H. Ford, Phys.Rev.D46, 4510 (1993).

[16] K.K.Venkataratnam and P.K.Suresh Int.J.Mod.Phys.D13,239 (2004).

[17] N. D. Birrell and P. C. W. Davies, Quantum Field Theory in Curved Space (Cambridge University Press, 1982).

[18] C. M. Caves and D. F. Wall, Phys. Rev.Lett.57, 2164 (1980).

[19] J. R. Klauder and B. S. Skagersterm, Coherent States (World Scientific, 1985).

[20] B. L. Schumaker, Phys.Rep.135, 317 (1986). 